\title{
El Sistema de Información de Marketing (SIM) como estrategia competitiva de comunicación en las mipymes de México: estudio diagnóstico del impacto de las tic
}

\section{Marketing Information System (SIM) as a competitive communication strategy in Mexico's mipymes: impact of tics diagnostic study}

Emigdio Larios-Gómez (México)

herr.larios@gmail.com

Doctorado en Ciencias Administrativas Universidad de Guanajuato Campus Celaya-Salvatierra
Héctor Cuevas-Vargas (México)

hcuevas@utsoe.edu.mx

$\mathrm{PhD}$ in Management Sciences Universidad Tecnológica del Suroeste de Guanajuato

Salvador Estrada Rodríguez (México)

salvador.estrada@gmail.com

Doctor en Economía, Gestión de la Innovación y Política Tecnológica

Universidad de Guanajuato Campus Celaya-Salvatierra

22 DE MARZO DE 2016

25 DE ABRIL DE 2016

27 DE MAYO DE 2016
Para citar este artículo / to Cite this article Larios-Gómez, E., Cuevas Vargas, H. \& Estrada Rodríguez, S., (2016), El sistema de información de marketing (SIM) como estrategia competitiva de comunicación en las mipymes de México: estudio diagnóstico del impacto de las TIC, Poliantea 12(23), pág. 129-163. 


\section{- El Sistema de Información de Marketing... - Emigdio Larios-Gómez, Héctor Cuevas-Vargas, Salvador Estrada R.}

\section{Resumen}

Se trata de una investigación empírica desde el enfoque cuantitativo de tipo transversal, no experimental, basada en el Modelo de Probst, de una muestra por conveniencia de 143 mipymes (micro, pequeñas y medianas empresas), ubicadas en la ciudad de Celaya, Guanajuato y de una muestra por conveniencia de 305 mipymes en la ciudad de Puebla. El objetivo del presente fue analizar comparativamente la competitividad de la existencia de un Sistema de Información de Marketing (SIM), creado y gestionado por las mipymes celayenses y poblanas. El estudio y análisis se realiza desde la perspectiva de la gestión del conocimiento y su papel esencial en el paradigma económico emergente y factor competitivo empresarial. El estudio revela un comportamiento similar entre las mipymes celayenses y poblanas, quienes no gestionan el conocimiento de marketing generado a través de sus sistemas de tecnología para la comunicación e información en la organización y presentan un grado bajo o nulo uso de las TIC y su medición de los conocimientos acumulados interna y externamente, lo que provoca una problemática de comunicación en los aspectos de marketing, al no contar con un SIM formal y estructurado, es decir uso de las TIC, a través de un CRM (Costumer Relationship Manager). Las mipymes poblanas y celayenses, en términos generales, no toman como importante el conocimiento generado en la organización, y las mipymes que presentan un grado bajo en la gestión del conocimiento, en relación

\section{Summary}

This is an empirical research from a nonexperimental transversal type quantitative approach, based on Probst Model, from a convenience sample of 143 micro and small enterprises (micro, small and medium enterprises), located in Celaya city, Guanajuato, and a convenience sample of 305 Mipymes (SMEs) in Puebla city. The goal was to comparatively analyze a Marketing Information System (SIM) competitiveness, created and managed by Celaya and Puebla's Mipymes. Study and analysis is done from a knowledge management perspective, and its essential role in the emerging economic paradigm and competitive business factor. The study reveals a similar behavior among Celaya and Puebla's Mipymes, who do not manage marketing knowledge generated through their organization communication and information technology systems, have a low or null use of TICS and its internally and externally accumulated measurement of knowledge, which causes communication problems in marketing aspects, not having a formal and structured SIM, i.e. use of TICS, through a CRM /Costumer Relationship Manager). In general terms, Puebla and Celaya's Mipymes do not take organization generated knowledge as important, and Mipymes that have a knowledge management low degree, in relation to their own competitiveness in market, do so without measuring or evaluating the obtained results with strategic planning and communication. The advantages of adopting Information and Communication Technologies (TIC) in organizations are becoming increasingly evident, whether from basic soffware use (Excel) 
con su competitividad en el mercado, lo hacen sin medir o evaluar los resultados obtenidos con la planeación estratégica y de comunicación de estas. Cada vez son más evidentes las ventajas al adoptar Tecnologías de la Información y la Comunicación (TIC) en las organizaciones, ya sea desde uso de software básico (Excel), hasta el sofisticado como un CRM; sin embargo, las micro, pequeñas y medianas empresas no están comprometidas con esto, por la carencia de una cultura organizacional, la escasez de recursos financieros, humanos y tecnológicos.

Palabras clave: gestión de marketing, gestión del conocimiento, competitividad, mipymes, CRM, Sistema de Información de Marketing (SIM). to sophisticated software such as a CRM; However, micro, small and medium enterprises are not committed to this, due to the lack of an organizational culture, the scarcity of financial, human and technological resources.
Key Words: marketing management, knowledge management, competitiveness, Mipymes, CRM, Marketing Information System (SIM). 


\section{Introducción}

En años recientes, la competitividad se ha vuelto la prioridad de gobiernos, objeto de estudio de instituciones académicas, y preocupación de inversionistas y empresarios (CEPAL, 2011). Existen instituciones, nacionales y extranjeras, que han desarrollado y publicado indicadores para tratar de medir la competitividad de los distintos países. El Foro Económico Mundial (WEF - The World Economic Forum), al igual que el IMCO (Instituto Mexicano para la Competitividad), han desarrollado indicadores para medir la competitividad de los países. Para el WEF, la competitividad es la habilidad que tienen los países para proveer altos niveles de prosperidad a sus ciudadanos. Para el IMCO, la competitividad significa la habilidad de una región para atraer y retener inversiones. A pesar de sus diferencias conceptuales, ambos índices utilizan variables sobre las instituciones y el ambiente de negocios (las empresas), en los países o estados para medir la competitividad de las mismas (IMCO, 2006). El índice de competitividad del Foro Económico Mundial incorpora variables como derechos de propiedad, independencia judicial, calidad de la infraestructura y cobertura educativa. La mayoría de las fuentes de información son instituciones públicas y una encuesta de empresarios. En el caso del IMCO, el índice de competitividad utiliza variables como corrupción, imparcialidad judicial, reforestación anual, acceso a servicios y población económicamente activa, entre otros. En este caso, la mayor parte de sus datos provienen de fuentes públicas. Estos indicadores sirven para que los gobiernos puedan implementar políticas públicas que 
atiendan los factores que inhiben su capacidad de atracción de inversiones y desarrollo. Creando una sana competencia entre gobiernos por mejorar sus políticas públicas y promover un mecanismo de desarrollo por imitación, en donde se identifiquen las mejores prácticas. A pesar de su utilidad, los índices de competitividad no ofrecen recomendaciones para desarrollar programas pro-activos de apoyo para ciertos sectores de la economía, como es el caso de las micro, pequeñas y medianas empresas.

Los niveles actuales de competencia local, regional, nacional e internacional han llevado a las empresas a desarrollar estrategias de mercado (mercadotecnia) cada vez más complejas (Muñoz y Montero, 2007), condición que impacta negativamente a la mipyme por sus niveles de ingreso y tipo de organización. Las empresas competitivas deben y saben cómo adaptarse a los mercados actuales y están conscientes de la importancia de tomar decisiones efectivas para lograr el desarrollo (Muñoz, 2003). Esto implica que las empresas deben tener la capacidad de moldear sus estructuras administrativas, financieras, operativas y comerciales, $y$ adecuar sus prácticas administrativas a las necesidades del entorno. En este mismo sentido, actualmente la competencia radica en la capacidad de la empresa para diseñar e implementar estrategias comerciales, pero desafortunadamente no todas cuentan con la información suficiente y experiencia para tomar decisiones efectivas; sobre todo si se habla de pequeños negocios, que por su poca participación en el comercio exterior, su acceso limitado a fuentes de financiamiento, desvinculación con los sectores más dinámicos, capacitación deficiente de sus recursos humanos, falta de vinculación con el sector académico, y necesidad de una cultura de innovación de procesos y desarrollo tecnológico, entre otras (Secretaría de Economía, 2006), limita su participación en el mercado, así como su práctica administrativa.

\section{La competitividad en la mipyme}

En estos últimos tiempos, las pymes latinoamericanas solo pueden sobrevivir en el proceso de desarrollo industrial si producen bienes y servicios específicos que no compitan con los productos de fabricación masiva de las grandes empresas industriales (OCDE, 2012). Típicamente, se trata 


\section{- El Sistema de Información de Marketing... - Emigdio Larios-Gómez, Héctor Cuevas-Vargas, Salvador Estrada R.}

de productos diseñados de acuerdo con los requisitos de los clientes, pequeñas series para nichos de mercado o productos estrechamente relacionados con el servicio al cliente (instalación, adaptación específica a sus necesidades, mantenimiento y otros). Estas pymes también producen servicios para grandes empresas o complementan la oferta de servicios de estas. Actualmente, la especialización de la empresa impone mayores exigencias de calificación técnica y empresarial, siendo condición previa, en especial, la capacidad de innovar continuamente. La mayoría de las pymes en América Latina no cuenta con estas calificaciones. Casi todas estas empresas se insertan en áreas de producción estandarizadas y con baja intensidad de conocimientos, donde compiten directamente con la producción a gran escala y/o con grandes casas comerciales. Un ejemplo de lo anterior es la producción de bienes de consumo masivo (alimentos genéricos, calzado, confección), donde las pymes latinoamericanas no encuentran ventajas competitivas (Altenburg, T. y U. Eckhardt, 2006). Como se puede observar en la Figura 1, las pymes en países desarrollados funcionan de soporte y proveen a las grandes empresas y otras ofertan bienes y servicios personalizados para no competir con los masivos de las grandes. Caso contrario sucede en las pymes de países en desarrollo (como los latinoamericanos), donde la mayoría de las pymes compiten en mercados de productos masivo, acaparados por las grandes empresas, y el resultado de esto es un círculo vicioso en el que la baja productividad inicial y la ausencia de transferencia de conocimiento entre empresas se refuerzan mutuamente (Altenburg, 2011). 
Figura 1. Organización Industrial de las Pymes.

Típica organización industrial en países desarrollados

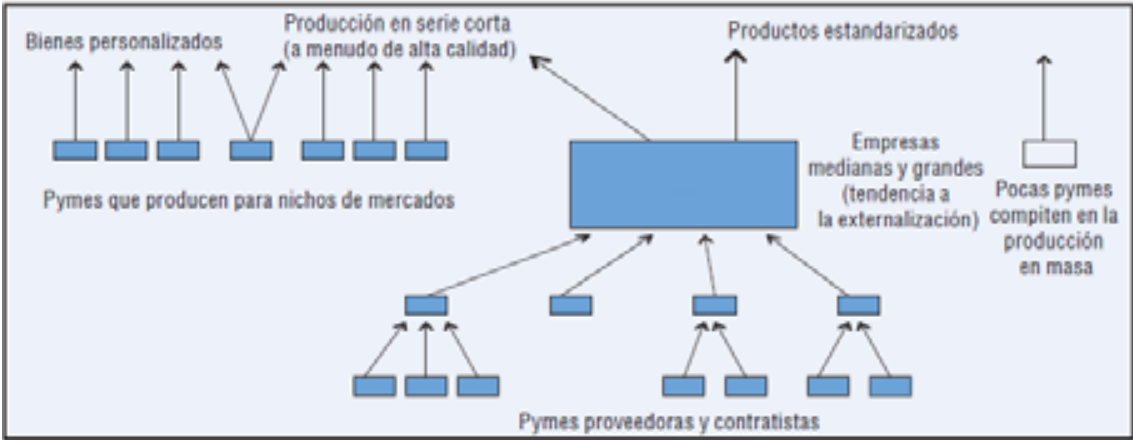

Típica organización industrial en países en desarrollo

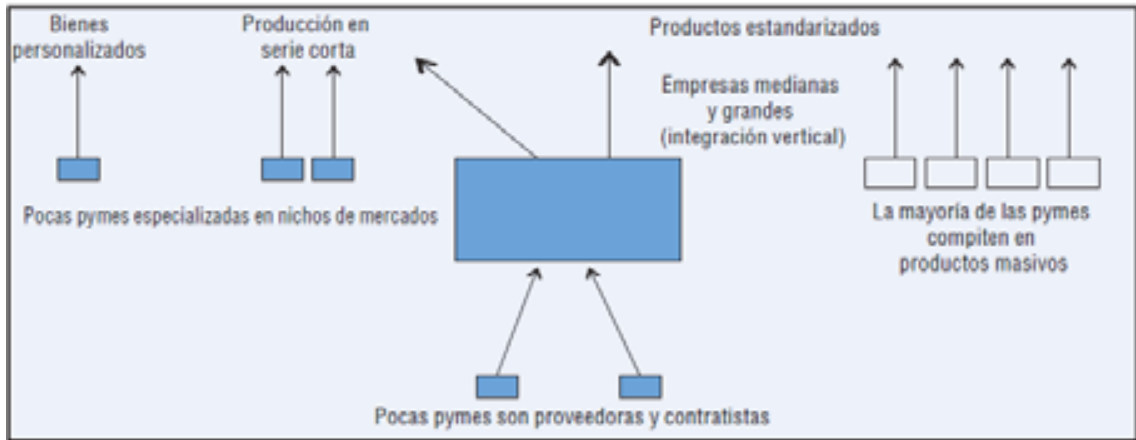

Fuente: Altenburg y Eckhardt (2006).

En México existen alrededor de cuatro millones de empresas; de estas, solo 6.700 son empresas grandes, el resto, las micro, pequeñas y medianas empresas representan $99,8 \%$, generan $52 \%$ del producto interno bruto (PIB) y contribuyen con $72 \%$ de los empleos formales. Las microempresas representan el 95\% de las empresas establecidas y el 38,3\% del empleo, muchas no generan ganancias o tienen niveles negativos de productividad y su contribución al PIB es muy débil, por lo que es necesario implementar una política que tenga como objetivo lograr que se reconozcan como negocios que deben generar riqueza. Por su parte, las pequeñas y medianas empresas representan el 4,8\% de las empresas del país y generan el $32,7 \%$ del empleo, son sumamente productivas y constituyen el eje de actividad económica nacional al 
ser los principales proveedores de las grandes empresas (OCDE y Fundación IDEA, 2010). A pesar de su importancia numérica, y a diferencia de las empresas de mayor tamaño, las mipymes mexicanas tienen que superar varios obstáculos para su desarrollo y sobrevivencia. Las mipymes se enfrentan a un difícil acceso al financiamiento, poca información sobre los mercados de sus productos, capital humano con poca capacitación, uso limitado de la tecnología e ignorancia acerca de los requisitos y normas para poder comercializar sus productos en mercados internacionales. Cabe resaltar que para la agrupación de las empresas -sujetos de estudio- se tomó en cuenta la Ley para el desarrollo de la competitividad de la micro, pequeña y mediana empresa (2015), que clasifica a la pyme por el número de trabajadores, el sector en el que opera y por el rango del monto de ventas anuales (ver Tabla 1).

Para definir la competitividad de las empresas, con base en lo mencionado por Pérez y Pérez (2008), la conceptualización de competitividad ha sufrido modificaciones tanto en su definición como en su contenido, de acuerdo con el panorama económico mundial, centrando su objetivo en los ámbitos nacional, sectorial y empresarial. Para este mismo autor, la competitividad es la capacidad que tiene una organización pública o privada de mantener ventajas que le permitan alcanzar y mejorar la posición socioeconómica ante otras empresas. Por otra parte, Cruz (2012), menciona que las empresas dedicadas a la producción de algún bien deben buscar la innovación para ser competitivas, sin embargo, muchas ven entorpecida esta labor por procesos de negocio y sistemas informáticos inadecuados que generan fallas de comunicación, errores laborales, retrasos y mayores costos.

Tabla 1. Clasificación de las mipymes en México

\begin{tabular}{cccc}
\hline \multicolumn{4}{c}{ Estratificación por Número de Trabajadores } \\
\hline Sector/Tamaño & Industrial & Comercio & Servicio \\
Micro & $0-10$ & $0-10$ & $0-10$ \\
Pequeña & $11-50$ & $11-30$ & $11-50$ \\
Medina & $51-250$ & $31-100$ & $51-100$ \\
\hline
\end{tabular}

Fuente: Ley para el desarrollo de la competitividad de la micro, pequeña y mediana empresa, 2015

De la Cruz, Morales y Carrasco (2006), sostienen que el desarrollo de capacidades en un sector y sus empresas contribuye a impulsar la competitividad de una gama de productos o servicios, y aquellas también influyen profundamente en las capacidades de crecimiento 
y diferenciación competitiva de una gama de productos mucho más allá que en el éxito o fracaso de un producto único. Solleiro y Castañón (2005), argumentan que la competitividad es un concepto complejo que se ha estudiado desde diversos enfoques y disciplinas. Señalan que el desempeño competitivo de la empresa depende en primera instancia de su capacidad para administrar los elementos internos que se encuentran bajo control; complementario a esto señalan que la competitividad también depende de la calidad de las interacciones que la empresa tiene establecidas con una serie de factores tanto internos como externos. En las aportaciones de este autor, dice que los elementos que influyen a la competitividad en las empresas son: la gestión exitosa de los flujos de producción, materias primas e inventarios, la gestión exitosa de los mecanismos de interacción entre planeación, mercadotecnia, investigación y desarrollo formal, diseño, ingeniería y producción industrial, la capacidad para combinar actividades de investigación y desarrollo e innovación en cooperación con universidades y otras empresas, la capacidad para incorporar definiciones más exactas de características de la demanda y evolución de mercados en el diseño y producción de estrategias, la capacidad para organizar con éxito relaciones inter-empresariales con proveedores y clientes, $y$ la mejora de las capacidades de los trabajadores mediante la inversión en entrenamiento especializado $y$ en la generación de altos niveles de responsabilidad en los trabajadores de producción. $\mathrm{Y}$ todas estas funciones deben ser concentradas en un sistema manual o electrónico que concentre toda la información disponible, principalmente en un CRM (Customer Relationship Management), que defina una metodología de trabajo para el manejo de la comunicación del negocio, que conjunte procesos y procedimientos, y que junto a un software de gestión de la comunicación interna y externa, se convierte una potente herramienta para la gestión de las relaciones con el cliente, proveedores y trabajadores, la cual aporta mucho valor a las empresas que lo implantan con sus tres pilares básicos: marketing, ventas y servicio postventa, ya que, en el contexto de la globalización de la comunicación, las tecnologías de información y la innovación, la pyme debe contar con los datos, información y 
aspectos indispensables del mercado, para mejorar la competitividad.

Quiroga (2003), propone un modelo para determinar la competitividad de la pyme, para lo cual determinó los factores y variables críticas que incorporan elementos internos controlables por las empresas, así como elementos externos que afectan su desempeño. Martínez, M., Sánchez, L., Santero, R. y Marcos, M. (2009) sugieren que los indicadores clave que inciden en la competitividad son los indicadores externos que tienen que ver con el entorno de los negocios, la tecnología e innovación que se mide en la capacidad para lanzar nuevos productos al mercado, la calidad, la gestión de los recursos humanos, las capacidades directivas en el uso de nuevas herramientas de gestión, la internacionalización -entendida como la capacidad para llegar a los mercados externos- y el financiamiento como una fuente necesaria para su permanencia. Con base en las aportaciones de los autores mencionados, se presenta en la Tabla 2, los indicadores propuestos, que se han tomado en cuenta para medir la competitividad de la empresa.

Actualmente, la empresa es considerada como un sujeto social (más que una identidad), y la intervención sistemática sobre la información se ha transformado en una necesidad impostergable para el crecimiento y para la permanencia de la pyme, no solo en países en desarrollo, sino también en todo los países emergentes. La comunicación es una herramienta importante, por medio de la cual las empresas intentan adaptarse y responder a los condicionamientos actuales (nuevos competidores, desarrollo de proveedores, nuevos consumidores y tendencias sectoriales). Por esto se torna importante intervenir en la pyme desde una perspectiva comunicacional integradora: el SIM. Por lo general, el conocimiento en las organizaciones (a través de la comunicación), carece de una estructura que facilite su utilización en forma efectiva; por otra parte, los conocimientos que se generan y apropian a través de procesos de competitividad e innovación, mediante la adquisición de tecnología, el trabajo con proveedores y clientes, las interacciones con los competidores y el entorno empresarial, presentan dificultades para ser integrados -en una base de datos- a las acciones y actividades cotidianas de la organización. Los nuevos paradigmas 
tecno-económicos que están generando una "tercera revolución industrial", aumentan la dependencia de los procesos de crecimiento y desarrollo económico de las capacidades para crear valor mediante la incorporación del conocimiento, la comunicación, la innovación y difusión del uso productivo de la tecnología (CEPAL, 2012). Por esta razón, es necesario desarrollar mecanismos que permitan a todas las personas disponer de todo el potencial del conocimiento que se encuentra disperso en la organización. Esto implica crear y desplegar una estrategia de gestión del conocimiento y la comunicación que integre cinco acciones fundamentales: entender necesidades y oportunidades del conocimiento; construir conocimientos relevantes para el negocio; organizar y distribuir el conocimiento de la organización; crear condiciones para la aplicación del conocimiento de la empresa, y explotar el conocimiento (Nagles G. y Nofal, 2007).

Tabla 2. Indicadores de Competitividad Empresarial

\begin{tabular}{|c|c|c|c|c|c|c|}
\hline \multicolumn{7}{|c|}{ Gadro 1. Indicadores de competitividad empresarial } \\
\hline Indicador/Autor & $\begin{array}{l}\text { Rubioy Aragón } \\
\text { (2006) }\end{array}$ & $\begin{array}{c}\text { De la Cruz, Moralesy } \\
\text { Carrasco (2006) }\end{array}$ & $\begin{array}{l}\text { Solleiroy Castanion } \\
\text { (2005) }\end{array}$ & $\begin{array}{l}\text { OCDE } \\
\text { (1992) }\end{array}$ & $\begin{array}{l}\text { Quiroga } \\
\text { (2003) }\end{array}$ & $\begin{array}{c}\text { Martinez, Santero, Sanchezy } \\
\text { Marcos (2009) }\end{array}$ \\
\hline Indicadores extemos & & & $x$ & & $x$ & $x$ \\
\hline Tecnologia & $x$ & $x$ & $x$ & & $x$ & $x$ \\
\hline Innoración & $x$ & & & & & $x$ \\
\hline Mercadotecnia & $x$ & $x$ & $x$ & $x$ & & \\
\hline Rexursos Humanos & $\mathrm{X}$ & $x$ & $\mathrm{X}$ & $x$ & $x$ & $x$ \\
\hline Capacidades directivas & $x$ & & & & $x$ & $x$ \\
\hline Recursos Finandieros & $x$ & $x$ & $x$ & & $x$ & \\
\hline Cultura & $x$ & & & & & \\
\hline Calidad & $x$ & & $x$ & & $x$ & $x$ \\
\hline Producción & & $x$ & & $x$ & $x$ & \\
\hline Logistica & & $x$ & & & & \\
\hline Organización interna & & & $x$ & & $x$ & \\
\hline Compras & & & $x$ & $x$ & $x$ & \\
\hline Investigación y desarrollo & & & $\mathrm{X}$ & $x$ & $x$ & $x$ \\
\hline Interacción con proveedores y dientes & & & & $x$ & & \\
\hline Internacionalización & & & & & & $x$ \\
\hline Finandiamiento & & & & & & $x$ \\
\hline
\end{tabular}

Fuente: Saavedra, Milla y Tapia (2013).

\section{La competitividad en las} organizaciones, desde la gestión del conocimiento, la comunicación y la mercadotecnia: el uso de las TIC

Para la presente investigación se ha tomado como base a Davenport y
Prusak (2001), quienes definen a la gestión del conocimiento como un proceso lógico, organizado y sistemático para producir, transferir y aplicar en situaciones concretas en una combinación armónica de saberes, experiencias, valores, información 


\section{- El Sistema de Información de Marketing... - Emigdio Larios-Gómez, Héctor Cuevas-Vargas, Salvador Estrada R.}

contextual y apreciaciones expertas que proporcionan un marco para su evaluación e incorporación de nuevas experiencias e información, explotando y desarrollando estos activos del conocimiento que posee la organización con el propósito de que esta sea capaz de cumplir con su misión estratégica. También se ha tomado en cuenta la definición de Nonaka y Takeuchi (1995), que describen a la gestión del conocimiento como el valor creado por una organización que es primariamente determinado por la transferencia tácita y explícita de conocimiento entre individuos y en la conversión del conocimiento de un tipo a otro. Solleiro y Castañón (2005), argumenta que el desempeño competitivo de la empresa depende en primera instancia de su capacidad para administrar los elementos internos que se encuentran bajo control, y de factores externos que no puede controlar. También señalan que los elementos que influyen en la competitividad de las empresas son: 1 . La gestión de los flujos de producción (materias primas e inventarios), 2. La gestión de los mecanismos de interacción-que incluye a la comunicación y sistemas de información- entre planeación, mercadotecnia, investigación y el desarrollo formal, diseño, ingeniería en la producción industrial, 3. La capacidad para combinar actividades de investigación y desarrollo e innovación en cooperación con universidades y otras empresas, 4. La capacidad para incorporar definiciones más exactas de características de la demanda y evolución de mercados en el diseño y producción de estrategias, 5. La capacidad para organizar con éxito relaciones inter-empresariales con proveedores y clientes y la mejora de las capacidades de los trabajadores por medio de la inversión en entrenamiento especializado y en la generación de altos niveles de responsabilidad en los trabajadores de producción. En lo que respecta a la competitividad de la comunicación en las mipymes, con base en las aportaciones de Dini y Stumpo (2011), existen dos características importantes que destacan en relación con la capacidad competitiva de este tipo de empresas, desde cómo gestionan su conocimiento adquirido mediante la comunicación interna y la relación de ese conocimiento-comunicación con su desempeño en el mercado (competidores, clientes y tendencias).

A pesar de las ventajas del uso de TIC en las organizaciones, en investigaciones realizadas, se observa 
que las pymes no realizan un uso y adopción comprometidos, debido a factores como la escasez de recursos financieros, la falta de recursos humanos capacitados y el acceso a los recursos tecnológicos, y sobre todo a la carencia de una cultura organizativa que incluya su uso (Dyerson y Harindranath, 2007). Las ventajas competitivas que brindan a una organización la implementación y uso de las TIC van desde aspectos del carácter operativo hasta del carácter estratégico. Desde el carácter operativo, el beneficio es el aumento de la eficiencia (la automatización de procedimientos, la clasificación de los productos, la disponibilidad de la información, entre otros), y desde el carácter estratégico, aporta a la mejora de los procesos empresariales (la gestión del conocimiento, establecimiento de alianzas estratégicas y la toma de decisiones oportuna y a tiempo). Las TIC posibilitan en el proceso productivo que los inputs se transformen en outputs, contribuyendo a introducir cambios en dichos procesos y contribuyendo a incrementar productividad en la organización (Billón, Lera y Ortiz, 2007). Las TIC constituyen uno de los elementos de gran impacto para cualquier entidad económica.
Su flexibilidad funcional y operativa son factores clave de éxito para el posicionamiento de cualquier institución (Aportela, 2007).

Los tipos de TIC para la pymes van desde las orientadas a la información (tecnologías para el almacenamiento y procesamiento de datos), como el datamining, los árboles de decisión y los sistemas integrados ERP (Enterprise Resource Planning) que permiten predecir y estimar comportamientos futuros que resultan muy útiles en el proceso de toma de decisiones estratégicas, siendo uno de ellos el CRM. Las TIC orientadas a la comunicación (reducen los costos de la comunicación y facilitan la transmisión de información) como la intranet, su diseño e implementación provee a la pyme una herramienta para la gestión del conocimiento y para la comunicación (interna y externa), que permite mejorar y agilizar la dinámica organizacional. Y las TIC orientadas a los flujos de trabajo (workflows), con las soluciones de software para las mesas de ayuda (helpdesk), para los diferentes roles de usuarios, clientes, gestores o administradores, en las que los usuarios o clientes pueden realizar solicitudes que involucran a 


\section{- El Sistema de Información de Marketing... - Emigdio Larios-Gómez, Héctor Cuevas-Vargas, Salvador Estrada R.}

diferentes áreas de la empresa, con la posibilidad de hacer seguimiento a cada una de las etapas del proceso de atención y servicio al cliente (tecnologías que posibilitan la integración de las diferentes capacidades empresariales para la obtención de resultados y la automatización de procesos).

De acuerdo con lo explicado en líneas anteriores, la competitividad de la empresa, desde la perspectiva de la gestión del conocimiento de las TIC en las organizaciones, es una disciplina estratégica de procesos inteligentes (lógicos, organizados, sistemáticos y tecnológicos), que promueve la generación, colaboración, interacción, transferencia y utilización del conocimiento explícito y tácito en la conversión de un expertise (mezcla de saberes, experiencias, valores e información de individuos), para el aprendizaje organizacional de una unidad económica, generándole nuevo valor de competitividad con miras a alcanzar sus objetivos con eficiencia y eficacia, con la capacidad de diseñar, crear, producir, ofrecer, distribuir y comercializar productos (bienes, servicios, ideas o experiencias) innovadores y de alto valor agregado -superior a los ofrecidos en el mercado- en las condiciones, cantidad y calidad que el cliente (presencial o virtual), nacional y/o internacional demanda (necesidades y deseos).

\section{Problema}

Los niveles actuales de competencia local, regional, nacional e internacional han llevado a las empresas a desarrollar estrategias de mercado cada vez más complejas (Muñoz y Montero, 2007), condición que impacta negativamente a la mipyme por sus niveles de ingreso y tipo de organización. Las empresas competitivas deben y saben cómo adaptarse a los mercados actuales y están conscientes de la importancia de tomar decisiones efectivas para lograr el desarrollo (Muñoz, 2003). Esto implica que las empresas deben tener la capacidad de moldear sus estructuras administrativas, financieras, operativas, de comunicación y comerciales, y adecuar sus prácticas administrativas a las necesidades del entorno. En este mismo sentido, actualmente la competencia radica en la capacidad de la empresa para diseñar e implementar estrategias, pero desafortunadamente no todas cuentan con la información suficiente y experiencia para tomar 
decisiones efectivas; sobre todo si se habla de pequeños negocios, que por su poca participación en el comercio exterior, su acceso limitado a fuentes de financiamiento, desvinculación con los sectores más dinámicos, capacitación deficiente de sus recursos humanos, falta de vinculación con el sector académico y necesidad de una cultura de innovación de procesos y desarrollo tecnológico, entre otras (Secretaría de Economía, 2006), limitan su participación en el mercado, así como su práctica administrativa.

En México se han realizado algunos estudios en las pymes desde una perspectiva de la gestión empresarial. Todos estos estudios han motivado el interés en la elaboración de la presente investigación desde una perspectiva de la información y la comunicación y sus bases tecnológicas de la mercadotecnia, como es el SIM o uso de un CRM, tema que no ha sido tratado por investigadores de las ciencias sociales y administrativas. Las estrategias de mercadotecnia más utilizadas en las organizaciones que inciden en la competitividad son las de calidad del producto y el manejo de precios, que sin conocer la información del mercado no podrían establecerse. El éxito competitivo se basa en cinco factores internos de las pequeñas y medianas empresas: a) recursos humanos, capacitados y preparados; b) planeación estratégica, corporativa e institucional; c) innovación en los procesos y formas de comunicación de la empresa; d) tecnología, uso de software y hardware y e) certificación de calidad, desde producción hasta la atención al cliente. Por su parte, Martínez, Charterina y Araujo (2010), identifican los factores de competitividad internos más importantes de las empresas industriales, como las capacidades directivas, las capacidades de innovación, las capacidades de marketing y las capacidades de calidad, las cuales se dan a través del uso eficiente de la información y comunicación de la empresa. Aunque estos autores no analizan directamente la mercadotecnia y la comunicación, en términos generales, sí mencionan que para que una empresa innove constantemente es indispensable el uso adecuado de estrategias de comunicación mercadológicas y que contribuyan a alcanzar el éxito competitivo.

El marketing puede ser utilizado por cualquier tipo de negocio, sin importar su ramo o tamaño. Sin embargo, la realidad es que las empresas 


\section{- El Sistema de Información de Marketing... - Emigdio Larios-Gómez, Héctor Cuevas-Vargas, Salvador Estrada R.}

micro o pequeñas no cuentan con recursos o experiencia para explotar la mercadotecnia en sus formas más sofisticadas; estas aplican el marketing en forma distinta y se basan en decisiones intuitivas. En un estudio realizado a pymes se concluyó que los problemas o factores que las afectan son: la falta de experiencia previa, bajo nivel de información técnica y profesional, poco desarrollo en habilidades de escritura, lectura, capacidad de escucha y de expresión, poca capacidad de identificar, diagnosticar y solucionar problemas, poca capacidad para manejar equipo destinado a procesar información (SIM o CRM), falta de iniciativa para tomar decisiones, poca capacidad para trabajar en equipo, falta de conocimientos, falta de inversión en capacitación. Y en los factores externos que limitan el desarrollo de las pymes, se encontró que son las condiciones de la competencia, el financiamiento del estado, la calidad y costo de la infraestructura, acceso al financiamiento, el sistema tributario, las dificultades para vender al sector público, el acceso a la tecnología, el acceso a mercados externos y el apoyo gubernamental, lo que se puede interpretar como una incapacidad de administrar el conocimiento a través de la comunicación. Por esto, en el presente estudio se plantea: ¿Cuáles el grado de gestión del conocimiento de comunicación en las mipymes poblanas y celayeses, en relación a su competitividad en el mercado? ¿Las mipymes en Puebla y en Celaya desarrollan la gestión del conocimiento e información, adquirido a través de la comunicación? ¿Pueden ser competitivas las mipymes mexicanas desde la perspectiva del marketing? ¿La gestión de la comunicación y el conocimiento desde la perspectiva de la mercadotecnia es un factor clave para la competitividad de las mipymes? ¿Cómo se gestiona el conocimiento de las TIC de marketing en las mipymes de Celaya-Guanajuato vs Puebla-Puebla, México?

\section{Método}

La presente se trata de una investigación de tipo concluyente con un enfoque cuantitativo de tipo transversal, no experimental y no probabilística (Kerlinger, 2002). Se trata de una investigación empírica basada en una encuesta, de una muestra por conveniencia de 143 empresas micro, pequeñas y medianas, de los sectores comercial, servicios, industrial, 
construcción y social, dedicadas a la comercialización al menudeo y mayoreo (retailing) ubicadas en la ciudad de Celaya, en el estado de Guanajuato, y de una muestra por conveniencia de 305 empresas micro, pequeñas y medianas, de la ciudad de Puebla, en el estado de Puebla, de los mismos sectores productivos que las celayenses. El objetivo del presente es identificar el conocimiento de las TIC de marketing creado por las mipymes (poblanas y celayenses) por medio de la transferencia tácita y explícita de conocimiento y la conversión del conocimiento tecnológico para la competitividad del mercado, tomando en cuenta su capacidad de producir, ofrecer y comercializar productos en las condiciones, cantidad y calidad que el mercado demanda. Para realizar el diagnóstico de los procesos de la gestión del conocimiento de las TIC, en las acciones de marketing de las mipymes sujeto de estudio, se tomó el modelo de ciclos de la gestión del conocimiento de varios autores (Dalkir, 2011; Wiig, 1993; McElroy, 1999; Rollet, 2003; Bukowitz y Williams, 2000; Meyer y Zack, 1996; Tannembaum y Alliger, 2000; Heisig, 1998 y Probst, Raub y Romhardt 2002). Como se muestra en la Tabla 3 , con base en un análisis semejante al realizado por Pérez-Soltero, Leal, Barceló-Valenzuela, León (2013), se seleccionaron los modelos estáticos de Rastogi (2000) y Tannembaum y Alliger (2000), y los modelos dinámicos de Heisig (1998) y Probst, Raub y Romhardt (2002). Finalmente, basado en el objetivo del presente estudio, se seleccionó el propuesto por Probst et al. (2002), porque contempla aspectos estratégicos de las organizaciones, entre ellos el uso de la tecnología, además de darle un mayor énfasis a la medición y valoración del conocimiento que sirve también de retroalimentación a la organización interesada en gestionar su conocimiento, para lo cual se adaptó el modelo desde la perspectiva de las TIC y el marketing. En la Figura 2 se muestra una adaptación del ciclo de la gestión del conocimiento de Probst et al. (2002).

Se han definido y adaptado las variables de estudio para la mipyme de Celaya, Guanajuato y la pyme de Puebla, Puebla, en un instrumento con 50 ítems, estructurado en siete secciones, correspondientes a la gestión del conocimiento de las TIC de la mercadotecnia. En la tabla 4 se definen cada una de las variables aplicadas. 
- El Sistema de Información de Marketing... - Emigdio Larios-Gómez, Héctor Cuevas-Vargas, Salvador Estrada R.

Tabla 3. Modelos integrales de la gestión del conocimiento.

\begin{tabular}{|l|l|l|l|}
\hline \multicolumn{2}{|c|}{ Modelos "estáticos” } & \multicolumn{2}{c|}{ Modelos “dinámicos” } \\
\hline Rastogi (2000) & Tannembaum & Heisig (1998) & Probst et al. (2002) \\
\hline - Identificación & y Alliger (2000) & & \\
- Rempartimiento & - Creación & - Identificación \\
- Captura & - Accesibilidad & - Almacenamiento & - Adquisición \\
- Adquisición & - Asimilación & - Distribución & - Desarrollo \\
- Almacenación & - Aplicación & - Distribución \\
- Compartimiento & & & - Utilización \\
- Aplicación & & & - Preservación \\
- Creación & & & \\
\hline
\end{tabular}

Fuente: Pérez-Soltero et al. (2013).

Figura 2. Gestión del Conocimiento de la Mercadotecnia.

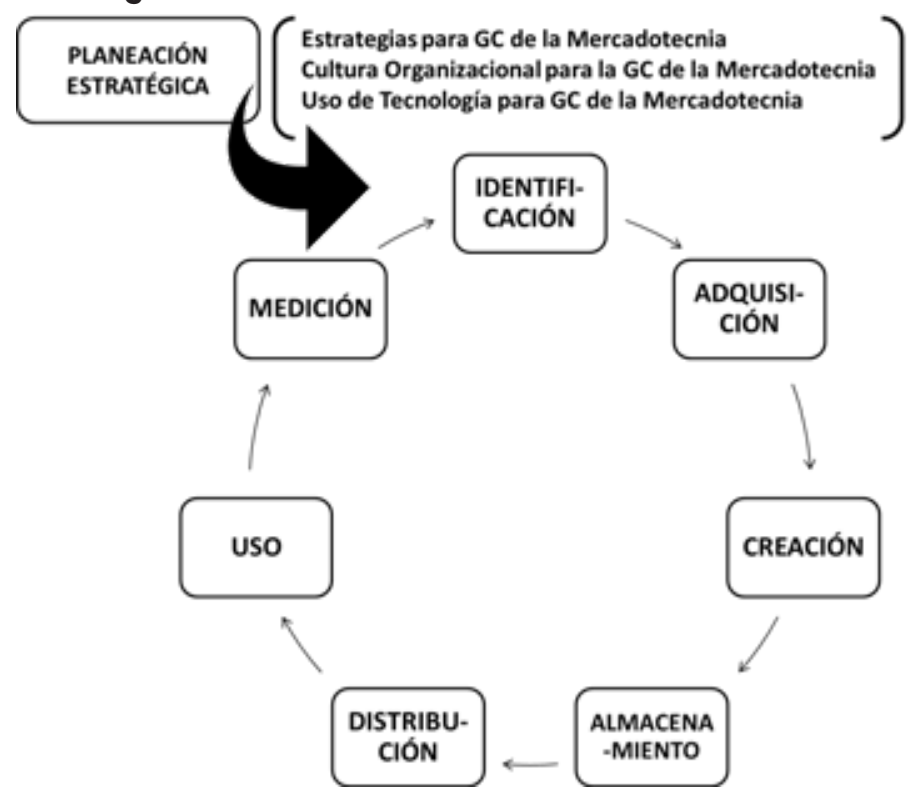

Fuente: elaboración propia con información de Probst et al. (2002). 
Tabla 4. Operación de Variables.

\begin{tabular}{|c|c|}
\hline Categoría & Descripción \\
\hline 1. Identificación & $\begin{array}{l}\text { Conocimientos, rol, disposición, medios } y \\
\text { conocimiento. }\end{array}$ \\
\hline 2. Adquisición & $\begin{array}{l}\text { Estrategias, fuentes internas, fuentes eternas, } \\
\text { capacitación y aprendizaje interno. }\end{array}$ \\
\hline 3. Creación & $\begin{array}{l}\text { Desarrollo, nuevas formas de trabajo, saber innovar, } \\
\text { programas de apoyo, eficacia en creación. }\end{array}$ \\
\hline 4. Almacenamiento & $\begin{array}{l}\text { Almacenar conocimiento y experiencia, acuerdos, } \\
\text { acceso, roles y registros del expertise. }\end{array}$ \\
\hline 5. Distribución & $\begin{array}{l}\text { Conocimiento personal, compartir, motivación y } \\
\text { sistema de distribución. }\end{array}$ \\
\hline 6. Uso & $\begin{array}{l}\text { Experiencias pasadas, motivación al uso, aplicación } \\
\text { de lo aprendido, ideas propias y de otros. }\end{array}$ \\
\hline 7. Medición & $\begin{array}{l}\text { Indicadores, medición y evaluación, nivel de } \\
\text { aprendizaje e instrumento de evaluación. }\end{array}$ \\
\hline
\end{tabular}

Fuente: elaboración propia con información de Probst et al. (2002).

\section{Resultados}

Los resultados fueron recolectados de la muestra, cabe resaltar que para a través de entrevistas con directo- la agrupación de las empresas -sujeres/dueños y gerentes/jefes de las tos de estudio- se tomó en cuenta la mipymes de Puebla y Celaya. Para Ley para el desarrollo de la compela fiabilidad de los siete constructitividad de la micro, pequeña y metos se realizó el cálculo de consisdiana empresa (2015), que clasifica a tencia interna basado en el Alfa de la pyme por el número de trabajadoCronbach. Para las mipymes de Ceres, el sector en el que opera y por el laya fue de 0,905 y para las de Puebla rango del monto de ventas anuales. fue de 0,806 (ambas determinadas como buenas), ver Tabla 5. La escala empleada fue de Likert, donde $1=$ Totalmente en desacuerdo y $5=$ Totalmente de acuerdo. Antes de exponer los resultados sobre la composición

Composición de la muestra. Celaya: la muestra estuvo compuesta por 143 mipymes de la ciudad de Celaya, donde el $29 \%$ fueron micro empresas (de 1 a 10 empleados), y el 54\% 
de ellas se dedican al comercio y el $34 \%$ a servicios. El $40 \%$ fueron pequeñas empresas (de 11 a 50 empleados), y en este grupo sobresale una pequeña diferencia en el número de empresas que se dedican al comercio, con el $39 \%$, y las que se dedica a los servicios, con el 33\%. El 31\% son medianas empresas (de 51 a 250 empleados), y el 33\% de estas se dedican a la industria, el $27 \%$ al comercio y el $18 \%$ a los servicios. En términos generales, el $41 \%$ de las mipymes pertenecen al sector comercial, $29 \%$ al de servicios, el $17 \%$ al sector industrial, el 5\% a la construcción y el $9 \%$ al sector social (Tabla 6).

\section{Composición de la muestra. Puebla:}

la muestra estuvo compuesta por 305 mipymes de la ciudad de Puebla y zonas conurbadas. El 36\% fueron micro empresas (de 1 a 10 empleados), y el $72 \%$ de ellas se dedican al Comercio. El 33\% fueron pequeñas empresa (de 11 a 50 empleados), y en este grupo sobresale que el $34 \%$ se dedica a los servicios. Finalmente, el $31 \%$ son medianas empresas (de 51 a 250 empleados), el $30 \%$ se dedican al comercio y el $28 \%$ a los servicios. En un análisis global, podemos observar que el $43 \%$ de las mipymes son del sector comercial, $24 \%$ de servicios, el 13\% de la industria, el 14\% de la construcción y el $6 \%$ a otros sectores-social (Tabla 7).

Tabla 5. Fiabilidad de las variables.

\begin{tabular}{|c|c|c|c|c|c|c|}
\hline \multirow[b]{2}{*}{ Variable } & \multicolumn{3}{|c|}{ CELAYA } & \multicolumn{3}{|c|}{ PUEBLA } \\
\hline & Media & $\begin{array}{c}\text { Alfa de } \\
\text { Cronbach }\end{array}$ & $\begin{array}{c}\text { Alfa de } \\
\text { Cronbach }\end{array}$ & Media & $\begin{array}{c}\text { Alfa de } \\
\text { Cronbach }\end{array}$ & $\begin{array}{c}\text { Alfa de } \\
\text { Cronbach }\end{array}$ \\
\hline 1. IDENTIFICACIÓN & 2.32 & 0.82 & \multirow{7}{*}{0.905} & 3.27 & 0.759 & \multirow{7}{*}{0.806} \\
\hline 2. ADQUISICIÓN & 2.47 & 0.95 & & 3.66 & 0.790 & \\
\hline 3. CREACIÓN & 2.72 & 0.98 & & 3.39 & 0.885 & \\
\hline 4. ALMACENAMIENTO & 2.97 & 0.88 & & 4.47 & 0.696 & \\
\hline 5. DISTRIBUCIÓN / DISEMINACIÓN & 3.32 & 0.83 & & 4.24 & 0.895 & \\
\hline 6. USO / APLICACIÓN & 3.56 & 0.98 & & 2.88 & 0.850 & \\
\hline 7. MEDICIÓN & 1.57 & 0.90 & & 3.06 & 0.769 & \\
\hline GLOBAL & 2.74 & & & 3.57 & & \\
\hline
\end{tabular}

Fuente: elaboración propia con SPSS 21. 
Tabla 6. Empresas de la muestra Celaya, según tamaño y sector.

\begin{tabular}{|c|c|c|c|c|c|c|c|}
\hline Tamaño & \multicolumn{7}{|c|}{ Sector } \\
\hline & Comercio & Servicios & Industrial & Construcción & Social & Tt no. & Tt \% \\
\hline Micro & $59 \%$ & $34 \%$ & $0 \%$ & $0 \%$ & $7 \%$ & $\mathbf{4 1}$ & $29 \%$ \\
\hline Pequeñas & $39 \%$ & $33 \%$ & $16 \%$ & $4 \%$ & $9 \%$ & $\mathbf{5 7}$ & $40 \%$ \\
\hline Medianas & $27 \%$ & $18 \%$ & $33 \%$ & $11 \%$ & $11 \%$ & $\mathbf{4 5}$ & $31 \%$ \\
\hline Total & $\mathbf{4 1 \%}$ & $\mathbf{2 9} \%$ & $\mathbf{1 7 \%}$ & $\mathbf{5 \%}$ & $\mathbf{9 \%}$ & $\mathbf{1 4 3}$ & $100 \%$ \\
\hline
\end{tabular}

Fuente: elaboración propia con SPSS 21

Tabla 7. Empresas de la muestra Puebla, según tamaño y sector.

\begin{tabular}{|c|c|c|c|c|c|c|c|}
\hline Tamaño & \multicolumn{7}{|c|}{ Sector } \\
\hline & Comercio & Servicios & Industrial & Construcción & Otros & Total & Total \\
\hline Micro & $72 \%$ & $11 \%$ & $1 \%$ & $11 \%$ & $5 \%$ & $\mathbf{1 0 9}$ & $100 \%$ \\
\hline Pequeñas & $25 \%$ & $34 \%$ & $21 \%$ & $15 \%$ & $6 \%$ & $\mathbf{1 0 2}$ & $100 \%$ \\
\hline Medianas & $30 \%$ & $28 \%$ & $19 \%$ & $16 \%$ & $7 \%$ & 94 & $100 \%$ \\
\hline Total & $\mathbf{4 3} \%$ & $\mathbf{2 4 \%}$ & $\mathbf{1 3 \%}$ & $\mathbf{1 4 \%}$ & $\mathbf{6 \%}$ & $\mathbf{3 0 5}$ & $100 \%$ \\
\hline
\end{tabular}

Fuente: elaboración propia con SPSS 21.

\section{Resultados de la gestión competitiva del conocimiento de las TIC de marketing en las mipymes de Celaya y Puebla}

\section{Identificación del conocimiento}

de las TIC de marketing. La identificación del conocimiento externo, que significa el análisis y la descripción del entorno del conocimiento de una empresa (Probst et al., 2002). Con base en la Tabla 8 (ver Figura 3), se puede observar que las mipymes de Celaya poseen un bajo nivel de identificación del conocimiento de las TIC de marketing, tanto del que se encuentra en su entorno, como el que está disponible o poseen sus empleados. En términos generales, la media fue de 2,32, donde el sector de servicios cuenta con una media de 4,06, muy por arriba del sector industrial y social con una media de 2,03, cada uno. En un análisis detallado, se encontró que la empresa mediana es la que más gestiona el conocimiento de las TIC de marketing a partir de su identificación (la media en el sector comercial es de 2,78, en el sector de servicios es de 4,67, en el sector industrial es de 3,0, en el sector de la construcción es de 2,30 y en el sector social es de 2,68). La empresa del sector de servicios es quien gestiona eficientemente el conocimiento de las TIC de marketing externo (con una media de 4,67), ya que lo exige 


\section{- El Sistema de Información de Marketing... - Emigdio Larios-Gómez, Héctor Cuevas-Vargas, Salvador Estrada R.}

la naturaleza de su producto intangible, el trato del empleado con el cliente y la heterogeneidad del proceso de producción del servicio. Permite el desarrollo e implementación de un sistema de información que concentre todos los datos, interacciones e información posible, sea con base en datos convencionales usando las herramientas de office o sofisticados con un EPR-CRM.

Para el caso de la mipyme poblana, se presenta una media global de 3,27, interpretando que no están de acuerdo ni en desacuerdo con que el conocimiento de la posición en el mercado, la rentabilidad y la satisfacción de los clientes puede generar información para la toma de decisiones en la empresa. De forma individual, para el sector de servicios, la media es de 4,70 , lo cual refleja la importancia de conocer a sus clientes un poco más, en comparación al sector industrial (media de 3,23 ), ya que se trata de productos intangibles. Y para las empresas del sector otros (social), la media fue más alta $(4,89)$, lo que inferimos se debe a la importancia de la razón de ser de este tipo de empresas (Tabla 9, ver Figura 4). La importancia de este constructo en las organizaciones es porque a partir de que se identifiquen los conocimientos de las TIC de marketing, se puede trazar estrategias que permitan "anclar" un SIM a estas, y se posibilite su uso dentro de la misma organización.

\section{Adquisición del conocimiento} de las TIC de marketing. Las organizaciones o entidades económicas (sean empresas grandes o pequeñas), adquieren información y conocimiento por medio de experiencias de empleados, socios, proveedores, competidores y de los mismos clientes, que deberán ser compiladas en sistemas de información (SIM). En investigaciones recientes, se ha detectado que las pymes raras veces aprovechan al máximo los conocimientos que van adquiriendo a lo largo del tiempo (Probst et al., 2001). Para el caso de las mipymes ubicadas en la ciudad de Celaya no gestionan la adquisición del conocimiento de las TIC de marketing, la media general es de 2,47, ver Tabla 8 (ver Figura 3). Davenport y Prusak (2001), señalan que el conocimiento adquirido no tiene que haber sido creado recientemente, solo debe ser nuevo para la organización. Es decir, se deben aprovechar el conocimiento existente en el entorno de la empresa y ser adaptada al 
contexto de ella, debiendo ser tratada con un cuidado considerable, ya que no todo el conocimiento disponible beneficia a las mipymes, sino es adaptado a los contextos propios. Las empresas del sector de servicios son las únicas que relativamente gestionan la adquisición del conocimiento disponible interna y externamente (media de 3,71). Y es nuevamente la empresa mediana del sector de servicios, quien con una media de $4,42 \mathrm{ob}-$ tiene un mayor nivel de adquisición del conocimiento, en relación con los demás sectores, haciendo uso de plataformas tecnológicas que le permiten adquirir el sentir de sus clientes, la observación de sus empleados y el comportamiento de sus competidores, con el uso de software libre de Datamining y CRM.

En la mipyme poblana, la media global es de 3,66, donde todos los sectores coinciden en la importancia de las alianzas estratégicas y de la generación de conocimiento en beneficio y cumplimiento de los objetivos empresariales (la media promedio de todos los sectores fue de 4,66). Contrariamente (Tabla 9, ver Figura 4), lo relacionado con adquirir conocimiento a través de la formación y capacitación de sus empleados es menos importante para los sectores de la industria y de la construcción, con una media de 2,45 y 2,30 , respectivamente.

\section{Creación del conocimiento de las} TIC de marketing. La creación del conocimiento, desde la perspectiva de la gestión del conocimiento, siempre comienza por el individuo o empleado en todos los niveles de la organización, donde la empresa para la creación de estrategias o conocimientos de marketing, debe motivar a los empleados o desarrollar ambientes para la espontaneidad y creación de este. Es la interacción dinámica entre conocimiento tácito y explícito (Dalkir, 2011). Es decir, los conocimientos tecnológicos con los que el personal ya cuenta y los conocimientos (procesos, bases de datos o uso de software) que se construyen en la organización. En la Tabla 8 (ver Figura 3), se puede observar que el sector de servicios es el que, de manera más recurrente, crea conocimiento tecnológico para la comunicación de marketing en la organización. Con una media de 3,61, las empresas que ofrecen servicios en Celaya buscan nuevas formas y estrategias de marketing, así como nuevos conocimientos con base en la experiencia diaria de sus funciones. Aunque de manera global la media es de 


\section{- El Sistema de Información de Marketing... - Emigdio Larios-Gómez, Héctor Cuevas-Vargas, Salvador Estrada R.}

2,72, representando un bajo interés de las mipymes en la creación de conocimiento tecnológico y de comunicación de marketing, la pequeña y mediana empresa del sector comercial sí se preocupan por esto (media de 4,89 y 4,98 , respectivamente); en el sector de servicios y en el sector social solo la mediana empresa (media de 4,8 y 4,74 , respectivamente).

La creación del conocimiento de las TIC de marketing en la mipyme poblana es dada a través de la interacción dinámica entre conocimiento tácito y explícito (Dalkir, 2011), la media fue de 3,39 , donde la investigación y desarrollo concentra las medias más bajas en este conjunto de variables. El sector otros (compuesto por mipymes con objetivo social), no le interesa la investigación y el desarrollo por medio de la administración del conocimiento, seguido del ramo comercial y de la construcción (media de 1,20 y 2,80 respectivamente). Solo a las empresas de los sectores de servicios e industrial les es indiferente, aunque este último tiene una media de 3,70 (Tabla 9, ver Figura 4), lo que significa que la interacción del conocimiento en la toma de decisiones, a través de la investigación, empieza a tomar relevancia. Cuando las empresas $u$ organizaciones enfrentan problemas para distribuir y colocar el conocimiento, es preciso considerar que este se transfiera mediante acciones entre y dentro de los grupos y equipos de trabajo de la organización o entre individuos.

\section{Almacenamiento del conocimien-} to de las TIC de marketing. En la gestión del conocimiento es importante retener y guardar percepciones y experiencias (del mercado, de los empleados y de los clientes), más allá del momento en que ocurren, de modo que puedan recuperarse después (Probst et al., 2002). El almacenamiento/mantenimiento del conocimiento (Tabla 8, ver Figura 3), en las mipymes de Celaya no se registra del todo en documentos, manuales o en sistemas electrónicos especializados (CRM, Microsoft Office o base de datos). La media es de 2,97, para lo cual las empresas del sector de servicios son las únicas que resguardan, almacenan y registran la información de marketing que se recaba en la operación diaria de la empresa. Las más cercanas a estas son las empresas del sector industrial. A pesar de que la micro, pequeña y mediana empresa (de todos los sectores analizados), está consciente y de acuerdo 
en que debe almacenarse el conocimiento de marketing (sector comercial, de servicios y social con media de 5,00), no saben ni cuentan con repositorios (medias de 1,00 a 3,00), desde los cuales los usuarios pueden acceder fácilmente a un conocimiento pertinente y en el momento que estos lo necesiten, siendo uno de los factores determinantes de éxito para la competitividad de la empresa (Peluffo et al., 2002).

En la mipyme poblana el almacenamiento de la información se realiza mediante del uso inteligente de las bases de datos y el diseño de manuales, catálogos y directorios. La media global es de 4,47 (todos los sectores), lo que refiere a la importancia del conocimiento hacia los usuarios finales. Solamente los sectores de la industria y la construcción obtienen medias por debajo de la global ( 3,56 y 3,45 respectivamente), y el resto mayor a 4 (Tabla 9, ver Figura 4).

\section{Transferencia y distribución del conocimiento de las TIC de marke-}

ting. En lo referente a la transferencia del conocimiento (diseminación), las organizaciones, en términos generales, enfrentan problemas para distribuir y colocar a disposición de sus miembros el conocimiento que ellos necesitan (Probst et al., 2002). Para el conocimiento de las TIC de marketing, la micro y pequeña empresa de los sectores comercial, industrial y de la construcción, desde su administración, interacción y medios no se transfiere el conocimiento de marketing de forma adecuada, ver Tabla 8 (ver Figura 3), sino que se transfiere mediante acciones personales hacia uno o varios grupos específicos de individuos (entre y dentro de los grupos y equipos de trabajo de la organización o entre individuos), por medios tecnológicos de comunicación, como el correo electrónico y las redes sociales, lo cual no se realiza tal cual en la mipyme del sector comercio y de la construcción (media de 2,49 y 2,96, respectivamente). En las mipymes del sector industrial y social (media de 3,27 y 3,82 , respectivamente), solo se limitan al uso de fax, mensajería y correos electrónicos (uso personal) desde un uso estándar y convencional, sin contar con medios formales de recolección, seguimiento y comunicación de la información de marketing. La empresa mediana del sector servicios es la única que cuenta con plataformas electrónicas donde se almacena y se transfiere la información de marketing a los diferentes 


\section{- El Sistema de Información de Marketing... - Emigdio Larios-Gómez, Héctor Cuevas-Vargas, Salvador Estrada R.}

individuos, para la toma de decisiones (media de 4,03), a través de un CRM-Customer Relationship Management, ya sea diseñado a las necesidades de la empresa o de paquetería comercial.

La aplicación/utilización del conocimiento de las TIC de marketing en la mipyme poblana se presenta en una media global de 4,24. Para todas las empresas, de todos los sectores, es importante asegurar que el conocimiento presente de la organización se aplique de manera productiva para su beneficio (Probst et al., 2002). Los estilos de dirección, las políticas y la cultura de la organización que inciden en la aplicación del nuevo conocimiento, permitiendo la transferencia de las mejores prácticas (internas y externas) y el share de ideas, experiencias y conocimientos (León et al., 2009). En este caso, solo el sector comercial es indiferente (medias de 3,45 en la transferencia y 3,40 en el share. Tabla 9, ver Figura 4). En los demás sectores encontramos una gran disposición a la transferencia del conocimiento a través de compartir las mejores prácticas (conocimiento tácito) y las mejores ideas/experiencias/conocimiento generadas por los empleados (conocimiento explícito).

\section{Uso y aplicación del conocimien-} to de las TIC de marketing. En los resultados correspondientes al uso y aplicación (utilización de las TIC) del conocimiento con el objetivo de asegurar que el conocimiento presente en una organización se aplique de manera productiva para su beneficio (Probst et al., 2002). Las mipymes del sector servicio de Celaya aplican el conocimiento de las TIC de marketing que identifican, generan y almacenan en sus sistemas de información de marketing; la media es de 4,65, la más alta de todos los sectores (Tabla 8 , ver Figura 3), si hacen uso de las plataformas tecnológicas, libres o comerciales con las que cuentan en la organización. El sector de servicios aplica el conocimiento para mejorar e innovar en la atención al cliente, con una media general de 4,46; la empresa mediana de este sector considera de suma importancia esta actividad (media de 5,00), la micro y la pequeña empresa, también, pero con mejor impacto (media de 3,70 y 4,67, respectivamente).

Respecto al uso del conocimiento de las TIC de marketing en la mipyme poblana, en términos generales, la media es de 2,88 , lo que refleja la baja importancia del uso del conocimiento en la creación de 
nuevos productos, solución de problemas, creación de marcas y desarrollo de inventos y patentes. Lo anterior nos permite inferir la baja capacidad para usar, transformar y optimizar el conocimiento identificado, almacenado y distribuido de la organización. Esto, principalmente para el sector comercial (media de 1,86), de la construcción (media de 2,78 ) y social (media de 2,75 ). El único sector que repunta en esta variables es el sector industrial con una media de 4,03 , pero obteniendo medias de indiferencias en creación de marcas y patentes $(3,60 \mathrm{y}$ 3,40 respectivamente (Tabla 9, ver Figura 4).

\section{Medición del conocimiento de} las TIC de marketing. Probst et al. (2002), afirman que la medición permite evaluar periódicamente el valor de las iniciativas asociadas con las prácticas de la gestión del conocimiento en relación con su aporte al desarrollo de la organización, el análisis de los resultados y los criterios de desempeño. Para el caso del marketing, la medición del uso de las TIC en el rendimiento organizacional constituye la pieza clave para identificar el incremento y desarrollo del capital intelectual de las organizaciones. La medición o valoración del conocimiento de las TIC de marketing en las mipymes de Celaya (media de 1,57), es baja y en algunas empresas nula (Tabla 8, ver Figura 3). Las empresas del sector social no realizan una evaluación o medición del impacto, beneficios y oportunidades que tiene el conocimiento de las TIC de marketing generado en el interior de sus estructuras (empleados, proveedores, socios y directivos), y al exterior con el mercado (competidores, clientes y consumidores): la media es de 1,00 , le siguen las empresas del sector comercio en la misma situación, con una media de 1,42, las del sector de la construcción con 1,54 y las del sector industrial con 1,83.

En la Tabla 9 y Figura 4 se puede observar lo correspondiente a la medición del conocimiento de las TIC de marketing (del rendimiento organizacional para identificar el incremento y desarrollo del capital intelectual en la organización). La media global fue 3,06, lo cual nos indica que no es importante para las mipymes poblanas la evaluación del conocimiento generado en la empresa, interna o externamente. Con base en Probst et al. (2002), es la esencia del cumplimiento de los objetivos del conocimiento y sus 
resultados, y permite analizar las di- $\quad$ se preocupa un poco más por meferencias existentes entre los resulta- dir los resultados obtenidos a través dos reales y las medidas ideales de de la gestión del conocimiento (merendimiento (Indejikian, 2002), lo dia de 3,93), caso contrario del seccual no está pasando en las empre- tor comercio (media de 1,37). sas analizadas. El sector industrial

Tabla 8. Gestión de las TIC de mercadotecnia en la mipyme de la ciudad de Celaya-Guanajuato

\begin{tabular}{|r|c|c|c|c|c|c|}
\cline { 2 - 7 } Variable & Comercio & Servicios & Industrial & Construcción & Social & Global \\
\hline 2. IDENTIFICACIÓN & 1.79 & 4.06 & 2.03 & 1.70 & 2.03 & $\mathbf{2 . 3 2}$ \\
\cline { 2 - 8 } 2. ADQUISICIÓN & 2.33 & 3.71 & 2.19 & 2.24 & 1.89 & $\mathbf{2 . 4 7}$ \\
\cline { 2 - 8 } 3. CREACIÓN & 2.54 & 3.61 & 1.81 & 1.93 & 3.70 & $\mathbf{2 . 7 2}$ \\
\cline { 2 - 8 } 4. ALMACENAMIENTO & 2.97 & 3.72 & 2.98 & 2.61 & 2.55 & $\mathbf{2 . 9 7}$ \\
\cline { 2 - 8 } & 2.49 & 4.03 & 3.27 & 2.96 & 3.82 & $\mathbf{3 . 3 2}$ \\
\cline { 2 - 8 } 6. USO / APLICACIÓN & 3.07 & 4.65 & 3.85 & 3.13 & 3.08 & $\mathbf{3 . 5 6}$ \\
\cline { 2 - 8 } 5. DISTRIBUCIÓN / DISEMINACIÓN & 1.42 & 1.83 & 2.07 & 1.54 & 1.00 & $\mathbf{1 . 5 7}$ \\
\hline
\end{tabular}

Fuente: elaboración propia

Figura 3. Gestión de las TIC de Mercadotecnia en la mipyme de la ciudad de Celaya-Guanajuato

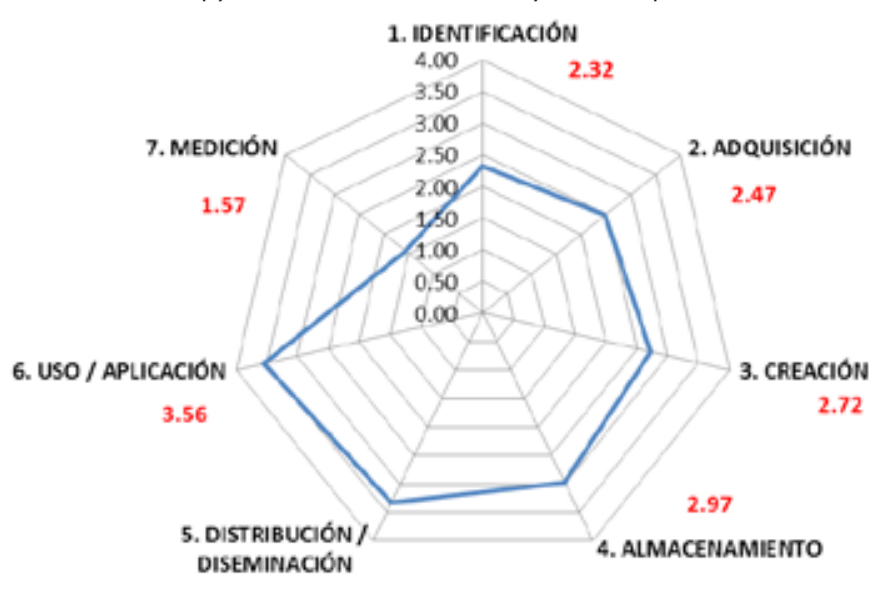

3.32

Fuente: elaboración propia. 
Tabla 9. Gestión de las TIC de mercadotecnia en la mipyme de la ciudad de Puebla-Puebla

\begin{tabular}{|c|c|c|c|c|c|c|}
\hline \multirow[b]{2}{*}{ Variable } & \multicolumn{6}{|c|}{ Sector } \\
\hline & Comercio & Servicios & Industrial & Construcción & Otros & Global \\
\hline 1. Identificación & 3.07 & 3.73 & 3.71 & 3.29 & 2.57 & 3.27 \\
\hline 2. Adquisición & 2.16 & 4.36 & 4.06 & 3.87 & 3.85 & 3.66 \\
\hline 3. Creación & 2.39 & 4.12 & 3.87 & 3.08 & 3.48 & 3.39 \\
\hline \multirow{2}{*}{ 4. Almacenamiento } & 4.76 & 4.94 & 4.17 & 4.00 & 4.49 & 4.47 \\
\hline & 3.43 & 4.53 & 4.79 & 3.80 & 4.65 & 4.24 \\
\hline 5. Distribución & 1.86 & 3.00 & 4.03 & 2.78 & 2.75 & 2.88 \\
\hline 6. Uso & 1.37 & 3.53 & 3.93 & 2.60 & 3.86 & 3.06 \\
\hline
\end{tabular}

Fuente: elaboración propia.

Figura 4. Gestión de las TIC de mercadotecnia en la mipyme de la ciudad de Puebla-Puebla

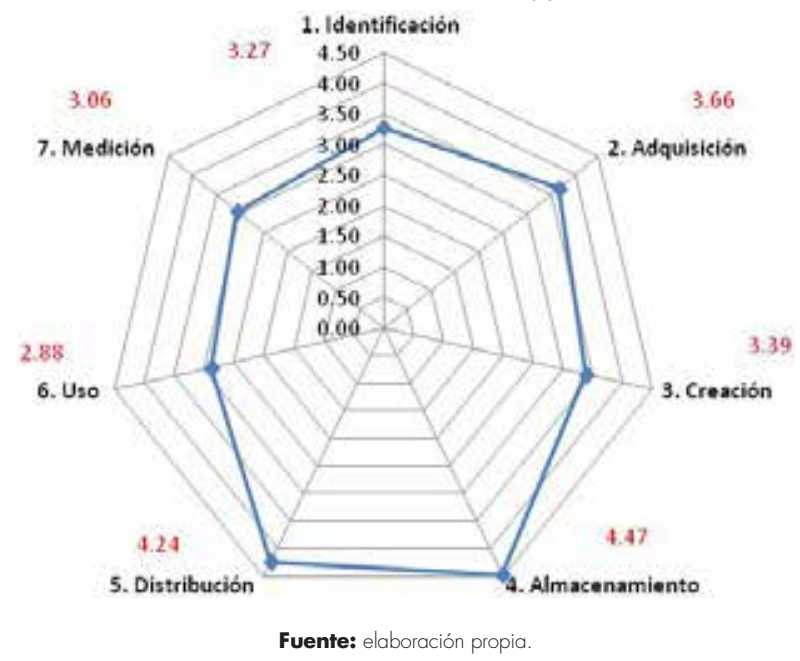

\section{Conclusiones}

Desde la propuesta señalada en el estratégica de procesos inteligentes presente, la gestión del conocimien- (lógicos, organizados y sistemáticos) to de las TIC de marketing en las or- ～-que no realiza la mipyme celayense, ganizaciones debe ser una disciplina ni poblana, con medias por debajo de 


\section{- El Sistema de Información de Marketing... - Emigdio Larios-Gómez, Héctor Cuevas-Vargas, Salvador Estrada R.}

2,72 en la identificación, adquisición y creación del conocimiento de marketing- que promueve la generación, colaboración, interacción, transferencia y utilización del conocimiento explícito y tácito en la conversión del expertise (mezcla de saberes, experiencias, valores e información de individuos) -lo cual no se da en las mipymes analizadas- para el aprendizaje organizacional de una unidad económica, generándole nuevo valor y de competitividad con miras a alcanzar sus objetivos con eficiencia y eficacia. En este sentido, las mipymes tanto de Celaya como de Puebla, del sector servicios, son las que más gestionan el conocimiento de las TIC de marketing (media promedio de 3,95).

Igualmente, con base en los resultados anteriores y respondiendo las preguntas planteadas en la presente investigación, podemos concluir que las mipymes poblanas y celayenses, en términos generales, no toman como importante el conocimiento generado en la organización, quizás por tratarse de micro, pequeñas y medianas empresas. Los dos únicos factores que obtuvieron el nivel más alto $(>4)$, fueron almacenamiento y distribución, acciones básicas de comercialización y en los sistemas de gestión de la calidad, que desde el año 2000 todas las empresas han implementado de en sus estructuras. Las mipymes poblanas presentan un grado bajo en la gestión del conocimiento, en relación con su competitividad en el mercado. Factores como creación de nuevos productos, solución de problemas, creación de marcas y desarrollo de inventos y patentes no son prioridad para estas empresas. Si lo reflexionamos por sector económico, notamos una ligera preocupación en las mipymes del sector industrial, ya que ponen en relieve la importancia de estos factores, como contribución a la dinámica de la innovación en la empresa, lo cual representa un gran atractivo, específicamente debido al bajo costo económico que implica la CGC en comparación con otros factores determinantes como son la I+D y la adquisición de equipo. No todas las mipymes en México desarrollan la gestión del conocimiento, o no todos los sectores económicos analizados, principalmente el comercial, el de construcción y el social-gubernamental (otros), que obtuvieron una media general $>2,72$ y $<3,66$. Los dos sectores con mayor desarrollo del conocimiento son el sector de servicios y el industrial. Por 
las características propias de estos, les es de importancia el gestionar las ideas, conocimientos, experiencias, tanto internas como externas, con el fin de cumplir con sus objetivos empresariales y comerciales.

Como se observó en los resultados presentados, tanto en Celaya como en Puebla, la mipyme carece de la capacidad de diseñar, crear, producir, ofrecer, distribuir y comercializar productos (bienes, servicios, ideas o experiencias) innovadores y de alto valor agregado superior a los ofrecidos en el mercado, en las condiciones, cantidad y calidad que el cliente (presencial o virtual, nacional y o internacional), demanda, desde una perspectiva de marketing. El marketing puede ser utilizado por cualquier tipo de negocio, sin importar su ramo o tamaño. Sin embargo, la realidad es que las empresas micro o pequeñas no cuentan con recursos o experiencia para explotar la mercadotecnia en sus formas más sofisticadas; estas aplican el marketing en forma distinta y se basan en decisiones intuitivas, lo que se reafirma con el presente estudio. Los empresarios, directivos y dueños de las mipymes deber ver a la mercadotecnia como factor clave para la competitividad de las empresas, ya que desarrollando una gestión del conocimiento de las acciones de marketing en la empresa, los procesos de intercambio de productos y valores (los cuales son generados mediante la gestión del conocimiento y experiencias de individuos) producirán efectos duraderos y significativos en la satisfacción de necesidades y deseos.

Las mipymes deben apropiarse de la mercadotecnia como una herramienta estratégica de la gestión de negocios (empresas, marcas, personas, lugares o productos), que le dará valor ante el mercado, cubriendo las bases mercadológicas de satisfacer necesidades, deseos y demandas, por medio de la comercialización (posicionamiento, intercambio y venta) de su producto nuevo o no (bien, servicio, idea o experiencia), en los diferentes canales de comercialización, sin inversiones altas, ya sea de forma directa o indirecta (presencial o virtual), porque recopila, analiza, sintetiza y gestiona la investigación de información y experiencias recolectadas de un mercado específico (clientes, consumidores, empleados, competidores, proveedores y socios) para la competitividad de la empresa (Larios-Gómez, 2013). 


\section{Referencias}

Altenburg, T. (2011), "Industrial Policy in Developing Countries. Overview and lessons from seven country cases", Discussion Paper 4/11, German Development Institute, Bonn.

Altenburg, T. y U. Eckhardt (2006), Productivity Enhancement and Equitable Development: Challenges for SME Development, Viena: UNIDO.

Aportela Rodríguez, I. M. (2007). Intranets: las tecnologías de información y comunicación en función de la organización. Acimed, 16(4), 8-15.

Billón Currás, M., Lera López, F. y Ortiz Serrano, S. (2007). Evidencias del impacto de las TIC en la productividad de la empresa, ¿fin de la«paradoja de la productividad»? Cuadernos de Economía: Spanish Journal of Economics and Finance, 30(82), 5-36.

Bukowitz, W. y Williams, R. (2000). The knowledge management fieldbook. London: Prentice Hall.

CEPAL (2011), Políticas para la innovación en las pequeñas y medianas empresas en América Latina, Marco, Dini y Giovanni Stumpo Compiladores.
CEPAL (2012), “Cambio estructural para la igualdad: una visión integrada de desarrollo", Trigésimo cuarto periodo de sesiones de la CEPAL, LC/G.2524 (SES.34/3), Naciones Unidas, Santiago.

Cruz, A. (2012), Pequeñas empresas. Innovación indispensable para empresas de manufactura, Recuperado de http://www.pymempresario. com/2012/02/innovacion- indispensable-para-empresas-demanufactura Dalkir, K. (2011). Knowledge management in theory and practice, USA: The MIT Press.

Davenport, T. H. y Prusak, L. (2001). Conocimiento en acción. Cómo las organizaciones manejan lo que saben. Buenos Aires: Prentice Hall.

De la Cruz, Morales y Carrasco (2006). Determinación de la competitividad en la Pyme Latinoamericana, Revista de investigación Institucional, p. 3. Referencia en Línea. http://www. alafec.unam.mx/docs/pymes.pdf

Dini, M. y G. Stumpo (coords.) (2004), Pequeñas y medianas empresas y eficiencia colectiva. Estudios de caso en América Latina, México, D.F: CEPAL-Siglo XXI Editores. 
Dyerson, R. y Harindranath, G. (2007). ICT Adoption \& Use by SMEs in the UK: A Survey of South East. Management of Engineering and Technology (1756-1770). Recuperado de http://ieeexplore.ieee.org/xpls/ abs_all.jsp?arnumber $=4349501$.

Heisig, P. (1998): Knowledge Management and Kaizen, Proceeding of the Second International EuroCINet Conference, Netherlands, 165-177.

IMCO (2006), Instituto Mexicano para la Competitividad, Situación de la Competitividad de México 2006: Punto de Inflexión, México: IMCO.

Indejikian, R. (2002). Performance evaluation and compensation research: An agency perspective. Accounting Horizons, 13(2), 147-57.

Kerlinger, (2002), Investigación del Comportamiento, McGraw Hill: México.

Larios-Gómez, E., (2013), Mercadotecnia de las instituciones de educación superior, Revista CPMark - Cuaderno Profesional de Marketing, 2(1) Recuperado de: http://docplayer.es/17269310-Cpmark-caderno-profissional-de-marketing-unimep-mercadotecnia-educativa-un- modelo-latinoamericano-emigdiolarios-gomez.html.

León, M.; Ponjuán, G.; Torres, D. (2009). Panorámica sobre la medición del conocimiento organizacional. ACIMED, 19(6).

Ley para el desarrollo de la competitividad de la micro, pequeña y mediana empresa (2015), Cámara de Diputados, México. Recuperado de: http:// www.diputados.gob.mx/LeyesBiblio/ pdf/247_210115.pdf.

Martínez, M., Sánchez, L., Santero, R. y Marcos, M. (2009). Factores de competitividad de la Pyme española 2008. España: Fundación EOI.

Martínez, R., Charterina, J. y Araujo, A. (2010). Un modelo causal de competitividad empresarial planteado desde la VBR: capacidades directivas, de innovación, marketing y calidad. Investigaciones Europeas de Dirección y Economía de la Empresa, 2(16), 165-188.

McElroy, M. (1999). The knowledge life cycle. The ICM Conference on KM. Miami.

Meyer, M. y Zack, M. (1996). The design and implementation of information products. Sloan. Management 


\section{- El Sistema de Información de Marketing... - Emigdio Larios-Gómez, Héctor Cuevas-Vargas, Salvador Estrada R.}

Review, 37(3), 43-59. http://dx.doi. org/10.3926/ic.381

Muñoz, D. y Montero N. (2007) Servicio a clientes $=$ un buen resultado. Revista Pyme, (163), 49-53

Muñoz, D. y Montero, N. (2003), Mercadotecnia una estrategia para competir. Adminístrate hoy, (107), 26-33.

Nagles G. y Nofal (2007). La gestión del conocimiento como fuente de innovación, Revista Escuela de Administración de Negocios, Recuperado de: http://www.redalyc.org/articulo. oa?id=20611495008

Nonaka, I. y Takeuchi, H. (1995). The knowledge creating company: How Japanese companies create the dynamics of innovation. New York: Oxford University Press.

OCDE (2012). Entrepreneurship at a Glance 2012. París: OCDE.

OECD y "Fundación IDEA" (2010) Consolidación de la competencia económica y la mejora regulatoria para la competitividad en México, estudio de caso. Puebla, prácticas y políticas exitosas para la mejora regulatoria y el emprendedurismo a nivel subnacional. México.
Peluffo, A. y Catalán, C. (2002). Introducción a la gestión del conocimiento y su aplicación al sector público. Gestión del conocimiento como base de una estrategia de desarrollo. Santiago de Chile.

Pérez, A. y Pérez, O. (2008). Competitividad y acumulación de capacidades tecnológicas en la industria manufacturera mexicana. Universidad Autónoma de Zacatecas. Recuperado de: http://redalyc.uaemex.mx/src/inicio/ ArtPdfRed.jsp?iCve=60111154005.

Pérez-Soltero, A.; Leal, V.; Barcelo, M.; Vanti, A. y Torres, C. (2013). Diagnóstico de los procesos de la gestión del conocimiento: caso de una empresa del sector restaurantero del noroeste de México, Produção em Foco, 1(1), 1-23.

Probst, G.; Raub S. y Romhardt, K. (2002). Administre el Conocimiento: Los Pilares del éxito. Traducido (del Inglés) por Núñez A. México: Pearson Educación.

Quiroga, D. (2003). Modelo matemático para determinar la competitividad de las Pymes. Cuadernos de Investigación y divulgación. Cali, Colombia: Corporación Universitaria Autónoma de Occidente. 
Ramaswamy, V., Gatignon, H. y Economía. Recuperado de http:// Reibstein, D. (1994). Competiti- www.economia.gob.mx/7p=7050. ve marketing behavior in industrial markets. Journal of Marketing, (58), 45-55.

Solleiro y Castañón (2005). Competitividad y sistemas de Innovación: los retos para la inserción de México en Rollet, H. (2003). Knowledge mael contexto global. Revista Iberoamenagement processes and technologies. Norwell, MA: Kluwer Academic Publishers. http://dx.doi. org/10.1007/978-1-4615-0345-3. ricana, 5, 15. Recuperado de http:// www.oei.es/salactsi/solleiro.pdf.

Tannembaum, S.I. y Alliger, J.M. (2000). Knowledge Management: ClaSaavedra, Milla y Tapia (2013). Derifying the Key Issues, UK: Ihrim.

terminación de la competitividad de la Pyme en el nivel micro: El caso del Distrito Federal, México.

Wiig, K. (1993). Knowledge management foundations. Arlington, TX: Schema Press.

Secretaría de Economía, (2006). Programa PIAPYME. Secretaría de 